\title{
Parcelamento e fontes de nitrogênio para produção de maxixe
}

\author{
Ademar P de Oliveira ${ }^{1}$; Flávio José V de Oliveira² ${ }^{2}$ Jandiê A da Silva²; Arnaldo Nonato P de Oliveira ${ }^{3}$; \\ Rodolfo R Santos ${ }^{3}$; Damiana F da Silva ${ }^{3}$ \\ ${ }^{1}$ UFPB-CCA, C. Postal 2, 58397-000 Areia-PB, Bolsista CNPq, ${ }^{2}$ UFPB, Pós-graduando em Agronomia; ${ }^{3}$ UFPB, Graduando em Agrono- \\ mia e Bolsista de Iniciação Científica, CNPq; ademar@cca.ufpb.br
}

\section{RESUMO}

Fontes e parcelamentos de nitrogênio foram avaliados no rendimento do maxixeiro, cv. Nordestino, na Universidade Federal da Paraíba, em blocos casualizados, esquema fatorial $2 \times 7$, com duas fontes de nitrogênio (uréia e sulfato de amônio) e sete épocas de aplicação: a) (100\% na semeadura; b) $100 \%$ aos 30 dias após a semeadura (DAS); c) $100 \%$ aos 60 DAS; d) $50 \%$ na semeadura e $50 \%$ aos 30 DAS; e) $50 \%$ na semeadura e $50 \%$ aos 60 DAS; f) $50 \%$ aos 30 e $50 \%$ aos 60 DAP; g) $33 \%$ na semeadura, $33 \%$ aos 30 e $33 \%$ aos $60 \mathrm{DAP}$, em quatro repetições. O peso médio de frutos foi inferior quando o sulfato de amônio foi fornecido $100 \%$ na semeadura e $100 \%$ aos $60 \mathrm{DAS}, 27$ e $21 \mathrm{~g}_{\text {fruto }^{-1}}$, respectivamente, não havendo alteração na fonte uréia, e entre as fontes. A produção de frutos por planta (2,364 kg planta $\left.{ }^{-1}\right)$, o número de frutos por planta (64 frutos planta $\left.^{-1}\right)$ e a produtividade de frutos $\left(20,93 \mathrm{t} \mathrm{ha}^{-1}\right)$ foram superiores significativamente quando o nitrogênio, fonte sulfato de amônio foi parcelado 50\% aos 30 e 50\% aos 60 DAS. Quando a fonte de nitrogênio foi a uréia, a produção de frutos por planta $\left(1,437 \mathrm{~kg}_{\text {planta }}{ }^{-1}\right)$, o número de frutos por planta (48 frutos planta $^{-1}$ ) e a produtividade de frutos $\left(12,66 \mathrm{t} \mathrm{ha}^{-1}\right)$ foram significativamente maiores quando foi parcelada $50 \%$ na semeadura e $50 \%$ aos 30 DAS.

Palavras-chave: Cucumis anguria L., uréia, sulfato de amônio, rendimento.

\author{
ABSTRACT \\ Parceling and nitrogen fertilization sources for gherkin \\ yield
}

The nitrogen sources and parceling were evaluated concerning to gherkin plant, Nordestino cv., in the Universidade Federal da Paraíba, Brazil. The experimental design was the randomized block design in the factorial scheme $2 \times 7$, constituted of two nitrogen sources (urea and ammonium sulfate) and seven application times: a) (100\% at sowing date; b) 100\% 30 days after sowing (DAS); c) 100\% 60 DAS; d) $50 \%$ at sowing date and $50 \%$ at $30 \mathrm{DAS}$; e) $50 \%$ at sowing date and $50 \%$ at $60 \mathrm{DAS}$; f) $50 \%$ at 30 and $50 \%$ at 60 DAP; g) $33 \%$ at sowing date, $33 \%$ at $3033 \%$ at $60 \mathrm{DAP}$, with four replicates. The average weight of the fruits was inferior, 27 and $21 \mathrm{~g}$, respectively, when the ammonium sulfate was $100 \%$ supplied at sowing and $100 \%$ at $60 \mathrm{DAS}$, as no alteration occurring in the urea source nor between those $\mathrm{N}$ sources. The yield of fruits of one plant $\left(2.364 \mathrm{~kg} \mathrm{plant}^{-1}\right)$, the number of fruits of one plant ( 64 fruits plant ${ }^{-1}$ ) and fruit productivity (20.93 $\mathrm{tha}^{-1}$ ) were significantly higher, when the nitrogen (ammonium sulfate source) was $50 \%$ parceled at 30 and $50 \%$ at 60 DAS. When urea was the nitrogen source, the yield of fruits of one plant (1.437 $\left.\mathrm{kg} \mathrm{plant}^{-1}\right)$, the number of fruits of one plant $\left(48\right.$ fruits plant $\left.^{-1}\right)$ and the fruit productivity $\left(12.66 \mathrm{tha}^{-1}\right)$ were significantly higher, when it was parceled $50 \%$ at sowing date and $50 \%$ at $30 \mathrm{DAE}$.

Keywords: Cucumis anguria L., urea, ammonium sulfate, yield.

(Recebido para publicação em 25 de junho de 2009; aceito em 23 de abril de 2010) (Received on June 25, 2009; accepted on April 23, 2010)

$\mathrm{O}$ maxixe (Cucumis anguria L.) é uma hortaliça de origem africana, bastante cultivada nas regiões Norte e Nordeste. Produz frutos sem sabor amargo e com variações quanto a espiculosidade e ao tamanho, geralmente com peso médio de $30 \mathrm{~g}$ (Modolo \&Costa, 2003). É comum encontrar plantas de maxixe crescendo de modo subespontâneo no meio de outras plantações, cuja produção atende ao consumo doméstico e ao mercado, quando há demanda.

O maxixe é utilizado na forma de fruto imaturo, podendo ser consumido in natura (salada), em conserva (picles) ou cozido (refogados, sopas etc.). De modo geral, é uma hortaliça subutilizada como alimento, tanto no Brasil quanto no resto do mundo (Robinson \& Decker-Walters, 1997; Bates et al., 1999).
O maxixe é pouco exigente em solo, porém adapta-se melhor àqueles arenosos, leves e soltos. Quanto a sua fertilização, muitos produtores não realizam adubações, isso porque ele se beneficia de resíduos de nutrientes aplicados anteriormente. Não obstante, em solos pobres é recomendado o fornecimento de matéria orgânica, nitrogênio, fósforo e potássio (Pimentel, 1985; Filgueira, 2000).

O nitrogênio é o segundo nutriente mais exigido pelas hortaliças (Filgueira, 2000). Seu fornecimento via adubação funciona como complementação à capacidade de suprimento dos solos, geralmente com teores baixos desse nutriente, em relação às necessidades das plantas (Malavolta et al., 1990). Portanto, quando o nitrogênio no solo encontra-se em quantidades insuficientes para o suprimento das plantas, suas folhas ficam cloróticas, e produzem menos, mas se estiver em excesso, a planta vegeta excessivamente e produz menos frutos (Malavolta et al., 2002). As fontes nitrogenadas mais utilizadas na agricultura brasileira são a uréia e o sulfato de amônio (Barbosa Filho et al., 2004). A uréia apresenta $45 \%$ de nitrogênio e o sulfato de amônio com $21 \%$ de nitrogênio e $23 \%$ de enxofre (Malavolta et al., 2002). No Brasil, cerca de $52 \%$ do $\mathrm{N}$ consumido é na forma de uréia, 19\% como sulfato de amônio e 12,1\% como nitrato de amônio (Sangoi et al., 2003).

$\mathrm{Na}$ adubação nitrogenada em hortaliças, incluindo aquelas produtoras de frutos, deve-se levar em consideração 
as exigências da cultura, condições de clima, além da idade da planta, pois a cultura precisa de níveis diferentes do nitrogênio que vai depender do seu estádio de desenvolvimento (Malavolta et al., 1997).

De acordo com Silva et al. (2004), uma das formas para reduzir perdas de nitrogênio e melhorar seu aproveitamento seria sua aplicação na semeadura ou transplantio juntamente com o fósforo e o potássio, e o restante distribuído em cobertura, em uma ou mais vezes, coincidindo com o período de maior exigência de cultura.

Nesse sentido, o parcelamento do nitrogênio pode amenizar as perdas, além de favorecer uma melhor produção devido ao eficiente aproveitamento do nutriente pelas plantas, devendo o mesmo ser aplicado na época de maior exigência pelas plantas, pois o nitrogênio que não é absorvido é perdido de alguma forma, seja por lixiviação ou volatilização (Barbosa Filho et al., 2004).

Pimentel (1985) recomenda o fornecimento do todo o nitrogênio (uréia) na semeadura, e Filgueira (2000) relata que esse nutriente deve ser fornecido na semeadura, e em adubação de cobertura.

Para o maxixeiro, pouco se conhece sobre suas exigências nutricionais. Até o momento, as recomendações de adubação para seu cultivo são feitas, com base em informações para as regiões Sul e Norte. Como todas as hortaliças, principalmente as produtoras de frutos, a adubação nitrogenada e seu manejo, são importantes para incrementar sua produção. Nesse sentido, informações sobre a melhor fonte de nitrogênio e o parcelamento mais adequado, poderão se converter num forte aliado para $o$ sucesso no seu cultivo.

Devido à escassez de informações para o manejo de adubação nitrogenada no maxixeiro, conduziu-se este trabalho com o objetivo de avaliar a sua resposta a fontes e parcelamentos de nitrogênio.

\section{MATERIAL E MÉTODOS}

O experimento foi realizado de abril a agosto de 2007, conduzido em condições de campo, na Universidade Federal da Paraíba. Pela classificação de Köppen, o clima do local da pesquisa é do tipo AS'(quente e úmido), precipitação pluviométrica média anual em torno de $1.400 \mathrm{~mm}$ e temperatura média anual entre $23^{\circ}$ e $24^{\circ} \mathrm{C}$, com Latitude $6^{\circ} 58^{\prime} 12^{\prime \prime}$ $\mathrm{S}$, longitude $35^{\circ} 42^{\prime} 15^{\prime}$ ' W. e altitude de $574,62 \mathrm{~m}$ ). A temperatura média em ${ }^{\circ} \mathrm{C}$, a precipitação pluviométrica em mm e a umidade relativa em \% do período experimental foram, respectivamente: abril = 24,$6 ; 211,7 ; 84 ;$ maio $=22,4 ; 151 ; 89$; junho $=23,5 ; 285,9 ; 92$; julho $=20,6$; 158,$3 ; 91 ;$ e agosto $=20,2 ; 156,8 ; 88$.

O solo da área experimental foi classificado de acordo com Embrapa (1999), como NEOSSOLO REGOLITICO Psamítico típico, textura franca-arenosa, com relevo local suave ondulado e regional forte ondulado e fase floresta subperenifólia, com as seguintes características química e física: $\mathrm{pH}=6,9$; $\mathrm{P}$ $=11,24 \mathrm{mg} \mathrm{dm}^{-3} ; \mathrm{K}=54,12 \mathrm{mg} \mathrm{dm}^{-3}$; $\mathrm{Al}^{+3}=0,00 \mathrm{cmol}_{\mathrm{c}} \mathrm{dm}^{-3} ; \mathrm{Ca}^{2+}=3,35 \mathrm{cmol}_{\mathrm{c}}$ $\mathrm{dm}^{-3} ; \mathrm{Mg}^{2+}=0,65 \mathrm{cmol}_{\mathrm{c}} \mathrm{dm}^{-3} ; \mathrm{Na}^{+}=0,07$ cmol dm ${ }^{-3} ; \mathrm{H}^{+}+\mathrm{Al}^{+3}=2,56 \mathrm{cmol} \mathrm{dm}^{-3}$; $\mathrm{SB}=4,21$; $\mathrm{CTC}=6,77$ e matéria orgânica $=24,93 \mathrm{~g} \mathrm{~kg}^{-1}$. A análise do solo foi realizada segundo metodologia descrita por Embrapa (1997); areia $=841,50 \mathrm{~g}$ $\mathrm{kg}^{-1}$; silte $=88,00 \mathrm{~g} \mathrm{~kg}^{-1}$; argila $=70,50$ $\mathrm{g} \mathrm{kg}^{-1}$; densidade global $=1,37 \mathrm{~g} \mathrm{~cm}^{-3}$; densidade de partículas $=2,61 \mathrm{~g} \mathrm{dm}^{-3}$; e porosidade total $=0,47 \mathrm{~m}^{3} \mathrm{~m}^{-3}$. O preparo do solo constou de roçagem, capinas e abertura de covas de plantio.

$\mathrm{O}$ experimento foi conduzido em delineamento de blocos casualizados, distribuindo-se os tratamentos em esquema fatorial $2 \times 7$, com quatro repetições. $\mathrm{O}$ primeiro fator correspondeu às fontes de nitrogênio (sulfato de amônio e uréia) e no segundo os parcelamentos do $\mathrm{N}$, em diferentes proporções, sendo: a) $100 \%$ na semeadura; b) 50\% na semeadura, 50\% aos 30 dias após a semeadura (DAS); c) 50\% na semeadura e 50\% aos 60 DAS; d) $100 \%$ aos 30 DAS; e) $100 \%$ aos 60 DAS; f) $50 \%$ aos 30 e $50 \%$ aos 60 DAS; g) 33\% na semeadura, 33\% aos 30 e $33 \%$ aos 60 DAS. As parcelas continham 32 plantas espaçadas de 2,00 $\mathrm{m}$ entre fileiras e 1,00 m entre plantas. A instalação da cultura foi realizada por meio de semeadura direta, colocando-se quatro sementes por cova da cultivar Nordestino (Hortivale), realizando-se desbaste quinze dias após, para duas plantas por cova.

A adubação seguiu as recomen- dações do Laboratório de Química e Fertilidade de Solo da Universidade Federal da Paraíba, e consistiu da aplicação nas covas de $100 \mathrm{~kg} \mathrm{ha}^{-1}$ de $\mathrm{P}_{2} \mathrm{O}_{5}$ (superfostato triplo), $10 \mathrm{t} \mathrm{ha}^{-1}$ de esterco bovino e de $70 \mathrm{~kg} \mathrm{ha}^{-1}$ de $\mathrm{K}_{2} \mathrm{O}$ (cloreto de potássio), Em cobertura forneceu-se $80 \mathrm{~kg}$ de N, nas fontes e parcelamentos, conforme descrição no delineamento experimental.

Realizaram-se os tratos culturais normais para a cultura, incluindo irrigação por aspersão, com turno de rega de três vezes por semana, procurando fornecer quantidade de água suficiente para o bom desenvolvimento da cultura nos períodos de ausência de precipitação e capinas com auxílio de enxadas para manter a cultura sempre livre de plantas invasoras.

As colheitas, em número de oito, foram efetuadas a cada três dias, no período de 60 a 110 dias após a semeadura, quando os frutos se encontravam imaturos e com coloração verde intensa. Os frutos colhidos foram transportados para o galpão, para avaliação do peso médio de frutos, número e produção de frutos por planta e produtividade de frutos.

Os resultados foram submetidos às análises de variância, sendo as somas dos quadrados médios comparados pelo teste F (5\%). Para comparação das médias foi empregado o teste de agrupamento Scott-Knott, a 5\% de probabilidade, empregando-se o "software" SAEG (2000).

\section{RESULTADOS E DISCUSSÃO}

Houve interação significativa entre os parcelamentos e as fontes de $\mathrm{N}$ para o número e produção de frutos por planta e para produtividade, e efeito significativo independente $(\mathrm{p} \leq 0,05)$ do parcelamento e das fontes de $\mathrm{N}$, apenas para o peso médio de frutos. O peso médio de frutos foi inferior estatisticamente quando o sulfato de amônio foi fornecido $100 \%$ na semeadura e $100 \%$ aos 60 DAS, 27 e 21 $\mathrm{g} \mathrm{fruto}^{-1}$, respectivamente, não havendo alteração significativa em função da fonte uréia, e entre as fontes (Tabela 1). Independente das fontes de nitrogênio, todos os pesos médios de frutos foram superiores aquele verificado por Leal et al. (2000), 19,6 $\mathrm{g}_{\text {fruto }}{ }^{-1}$ para a cv. Maxixe do Norte, no sistema convencio- 
nal de produção, mas foram inferiores ao obtido por Modolo \& Costa (2000) que constataram peso médio de fruto de 37,4 g fruto $^{-1}$, também para um acesso de maxixe do tipo comum.

Os resultados obtidos para o peso médio de frutos quando foi usado o sulfato de amônio, indicam que parte desse nutriente deve ser fornecida após o maxixeiro apresentar área foliar definitiva, a exemplo do quiabeiro onde Oliveira et al. (2003) obtiveram elevação no peso de frutos com fornecimento de nitrogênio, fonte sulfato de amônio, quando as plantas já apresentavam flores e frutos. Possivelmente a presença do enxofre no sulfato de amônio, 23\%, (Malavolta et al., 2002), tenha contribuído também para elevação do peso médio dos frutos. De acordo com Alvarez et al. (2007), o equilíbrio entre o nitrogênio e o enxofre no solo e na planta é refletido no crescimento e no estado nutricional do vegetal, melhorando a produção das culturas. Feltrin et al. (2005) atribuíram o aumento na produção de frutos do tomateiro à presença do enxofre (18\%) na composição do sulfato de potássio.

A produção de frutos por planta $\left(2,364 \mathrm{~kg}\right.$ planta $\left.^{-1}\right)$, o número de frutos por planta (64 frutos planta ${ }^{-1}$ ) e a produtividade de frutos $\left(20,93 \mathrm{t} \mathrm{ha}^{-1}\right)$ foram superiores significativamente quando o nitrogênio, fonte sulfato de amônio, foi parcelado $50 \%$ aos 30 e $50 \%$ aos 60 DAS. Quando a fonte de nitrogênio foi a uréia, a produção de frutos por planta $\left(1,437 \mathrm{~kg} \mathrm{planta}^{-1}\right)$, o número de frutos por planta (48 frutos planta ${ }^{-1}$ ) e a produtividade de frutos $\left(12,66 \mathrm{t} \mathrm{ha}^{-1}\right)$ foram significativamente maiores quando foi parcelada $50 \%$ na semeadura e $50 \%$ aos 30 DAS (Tabela 1), porém com valores inferiores àqueles obtidos pelo uso do sulfato de amônio.

Independente da fonte de nitrogênio, a produção e o número de frutos por planta foram superiores aqueles verificados por Azevedo Filho \& Melo (2003) que obtiveram produção média de $1,47 \mathrm{~kg}$ planta $^{-1}$ e número médio de 41 frutos planta ${ }^{-1}$. As maiores produtividades obtidas, independente das fontes de nitrogênio (Tabela 1), superaram as produtividades médias para o estado do Maranhão, 8,0 e 16 t ha-1 (Martins, 1986) e para o estado de São Paulo, 12 t ha $^{-1}$
(Melo \& Trani, 1998).

As menores produções obtidas com o uso da uréia, possivelmente estão relacionadas com as perdas de $\mathrm{N}$ provocadas pelo excesso de precipitações ocorridas no período do experimento, o que pode ter proporcionado sua lixiviação prejudicando a sua disponibilidade ao maxixeiro. Melo \& Marques (2000), relatam que a adubação nitrogenada apresenta a inconveniência de ser facilmente lixiviada da solução do solo.

As altas produtividades obtidas nesse estudo podem ser atribuídas ao fato de que a maior eficiência no uso de nitrogênio nessa hortaliça é quando a mesma apresenta flores e frutos, caracterizando as fases de maior absorção do nutriente (Modolo \& Costa, 2003). Para a cultura do maxixe, altas produções são obtidas quando o nitrogênio for adicionado uma parte após o desbaste e outra no início da frutificação (Filgueira, 2000).

Como a colheita do maxixe tem início entre 55 e 60 DAS (Pimentel, 1985), esse fato pode justificar o aumento de produtividade quando $50 \%$ do nitrogênio, na forma de sulfato de amônio foi fornecido aos 30 e 60 DAS, e o aumento obtido quando o nitrogênio na forma de uréia foi aplicado $50 \%$ aos 30 e $50 \%$ aos 60 DAS. Esses resultados parecem indicar que a produtividade de frutos pode ser aumentada com aplicação de nitrogênio na forma de sulfato de amônio quando o maxixeiro já apresentar área foliar definitiva e início de frutificação.

Comparando-se os efeitos isolados das fontes sobre a produtividade de frutos, verificaram-se diferenças significativas (Tabela 1), com superioridade do sulfato de amônio. A superioridade dessa fonte de nitrogênio deve-se, possivelmente, à presença do enxofre na sua composição $(23 \%)$. O enxofre é um nutriente muito importante para a produção de proteínas e clorofila e é componente de alguns hormônios da planta, que participam na melhoria do crescimento das raízes e da produtividade (Mendonça \& Peixoto, 1991). Outra possível explicação para a superioridade do sulfato de amônio, pode ser o fato dessa fonte de nitrogênio ser absorvido na forma amoniacal (Novais et al., 2007), e o íon amônio por ser um cátion, é facilmente retido nas cargas eletronegativas das argilas e da matéria orgânica do solo, o que minimiza a suas perdas (Lopes, 2004). Isto parece indicar que essa fonte tendeu a concentrar-se em torno da região de aplicação do adubo, concordando com o que foi observado por Rodrigues \& Kiehl (1992).

Além disso, a superioridade do sulfato de amônio pode estar relacionada com a presença de amônio nas camadas subsuperficiais do solo, porque mesmo estando em pequenas concentrações pode ocorrer a movimentação do nitrogênio na forma amoniacal (Gonçalves et al., 2001; Oliveira, 2001). De acordo com Silva et al. (2000) o amônio pode difundir-se em sentido descendente, contribuindo para reduzir as perdas por volatilização, e em algumas situações, principalmente em solos onde há maior infiltração de água, esse fato favorece a presença de amônia, pois prolonga o tempo de disponibilidade de $\mathrm{N}$ para as plantas.

A inferioridade da uréia, mesmo contendo mais que o dobro de nitrogênio na sua composição (44\%), em relação ao sulfato de amônio, pode ser atribuída ao fato de não conter o íon amônio na sua formulação. Quando a uréia é aplicada no solo, é hidrolisada rapidamente produzindo esse íon, o qual passa para a forma nítrica, que pode perder-se por lixiviação, ou ainda transformar-se em gás amônia e assim perder-se para a atmosfera por volatilização. Sua lixiviação é mais intensa quando é aplicada em solos arenosos, e em condições de alta precipitação, características presentes nas condições deste estudo. Nesse sentido, caso não haja um equilíbrio entre a quantidade de uréia adicionada ao solo e a quantidade perdida anualmente, é provável que ocorra redução da capacidade do solo em fornecer nitrogênio para as culturas, promovendo redução progressiva da produtividade (Gonçalves et al., 2000).

\section{AGRADECIMENTOS}

Os autores agradecem ao $\mathrm{CNPq}$ pela concessão da bolsa de doutorado ao segundo autor, e aos funcionários da UFPB Francisco de Castro Azevedo, José Barbosa de Souza, Francisco Silva do Nascimento e Genival Gomes da 
Tabela 1. Peso médio de frutos (PMF), número de frutos por planta (NFP), produção de frutos por planta (PFP) e produtividade de frutos de maxixeiro (PF) em função de fontes e épocas de aplicação de nitrogênio (average weight of the fruits, number of fruits per plant, production of fruits plant ${ }^{-1}$ and productivity of gherkin fruits depending on sources and times of nitrogen application). Areia, CCA-UFPB, 2007.

\begin{tabular}{|c|c|c|c|c|c|c|c|}
\hline \multirow[b]{2}{*}{ Fonte } & \multicolumn{3}{|c|}{ Parcelamento (\%) } & \multirow{2}{*}{$\begin{array}{c}\text { PMF } \\
\left(\text { g fruto }^{-1}\right)\end{array}$} & \multirow[b]{2}{*}{ NFP } & \multirow{2}{*}{$\begin{array}{c}\text { PFP } \\
\left(\text { kg planta }^{-1}\right)\end{array}$} & \multirow{2}{*}{$\begin{array}{c}\text { PF } \\
\left(t \text { ha }^{-1}\right)\end{array}$} \\
\hline & $\begin{array}{c}\text { Semea- } \\
\text { dura }\end{array}$ & $\begin{array}{c}30 \\
\text { DAS }\end{array}$ & $\begin{array}{c}60 \\
\text { DAS }\end{array}$ & & & & \\
\hline \multirow{7}{*}{$\begin{array}{l}\text { Sulato } \\
\text { de } \\
\text { amônio }\end{array}$} & 100 & 0 & 0 & $27 \mathrm{~b}$ & $12 \mathrm{~d}$ & $0,527 \mathrm{e}$ & $5,20 \mathrm{~d}$ \\
\hline & 50 & 50 & 0 & $34 \mathrm{a}$ & $50 \mathrm{~b}$ & $1,630 \mathrm{~b}$ & $14,40 \mathrm{~b}$ \\
\hline & 50 & 0 & 50 & $33 \mathrm{a}$ & $27 \mathrm{c}$ & $0,916 \mathrm{~d}$ & $8,09 \mathrm{c}$ \\
\hline & 33 & 33 & 33 & $32 \mathrm{a}$ & $32 \mathrm{~b}$ & $1,524 \mathrm{~b}$ & $12,60 \mathrm{~b}$ \\
\hline & 0 & 100 & 0 & $32 \mathrm{a}$ & $36 \mathrm{c}$ & $1,223 \mathrm{c}$ & $10,80 \mathrm{~b}$ \\
\hline & 0 & 0 & 100 & $21 \mathrm{c}$ & $12 \mathrm{~d}$ & $0,260 \mathrm{e}$ & $2,30 \mathrm{e}$ \\
\hline & 0 & 50 & 50 & $33 \mathrm{a}$ & $64 \mathrm{a}$ & $2,364 \mathrm{a}$ & $20,93 \mathrm{a}$ \\
\hline \multirow[t]{4}{*}{ Média } & & & & $30,3 \mathrm{~A}$ & $33 \mathrm{~A}$ & $1,20 \mathrm{~A}$ & $10,61 \mathrm{~A}$ \\
\hline & 100 & 0 & 0 & $28 \mathrm{a}$ & $22 \mathrm{c}$ & $0,634 \mathrm{c}$ & $0,73 \mathrm{c}$ \\
\hline & 50 & 50 & 0 & $28 \mathrm{a}$ & $48 \mathrm{a}$ & $1,437 \mathrm{a}$ & $12,66 \mathrm{a}$ \\
\hline & 50 & 0 & 50 & $27 a$ & $23 \mathrm{c}$ & $0,672 \mathrm{c}$ & $5,23 \mathrm{c}$ \\
\hline \multirow[t]{4}{*}{ Uréia } & 33 & 33 & 33 & $29 a$ & $45 \mathrm{~b}$ & $1,031 \mathrm{~b}$ & $9,00 \mathrm{~b}$ \\
\hline & 0 & 100 & 0 & $27 a$ & $30 \mathrm{c}$ & $0,849 \mathrm{~b}$ & $4,20 \mathrm{c}$ \\
\hline & 0 & 0 & 100 & $23 \mathrm{a}$ & $19 \mathrm{c}$ & $0,461 \mathrm{c}$ & $4,10 \mathrm{c}$ \\
\hline & 0 & 50 & 50 & $32 \mathrm{a}$ & $44 \mathrm{~b}$ & $0,797 \mathrm{~b}$ & $7,44 \mathrm{~b}$ \\
\hline Média & & & & $27,7 \mathrm{~A}$ & $33 \mathrm{~A}$ & $0,84 \mathrm{~B}$ & $6,19 \mathrm{~B}$ \\
\hline $\mathrm{CV}(\%)$ & & & & 10,2 & 20,1 & 23,2 & 23,5 \\
\hline
\end{tabular}

Letras minúsculas comparam médias do parcelamento dentro de cada fonte. Letras maiúsculas comparam médias entre as fontes. Médias seguidas da mesma letra não diferem entre si pelo teste de agrupamento Scott Knott.

Silva que viabilizaram a execução dos trabalhos de campo.

\section{REFERÊNCIAS}

ALVAREZ VVH; KURIHARA CH; PEREIRA NF. 2007. Enxofre. In: NOVAIS RF; ALVAREZ VVH; BARROS NF; FONTES RLF; CANTARUTTI RB; NEVE JCL (Eds). Fertilidade do Solo. Viçosa: UFV. p. 595644.

AZEVEDO FILHO JA; MELO AMT. 2003. Caracterização agronômica de genótipos de maxixe. In: CONGRESSO BRASILEIRO DE OLERICULTURA, 43. Resumos... Recife: SOB (CD-ROM).

BARBOSA FILHO MP; FAGERIA NK; SILVA OF. 2004. Fontes e métodos de aplicação de nitrogênio em feijoeiro Irrigado submetido a três níveis de acidez do solo. Ciência e Agrotecnologia 28: 785-792.

BATES DM; ROBINSON RW; JEFREY C. 1999. Biology and utilization of the Cucurbitaceae. Ithaca: Cornel University Press. 485p.

EMBRAPA. Centro Nacional de Pesquisa de Solos. 1999. Sistema brasileiro de classificação de solos. $412 \mathrm{p}$.

EMBRAPA. Serviço Nacional de Levantamento e Conservação do Solo. 1997. Manual de métodos de análise de solo. Rio de Janeiro: Ministério da Agricultura, 212p.

FELTRIN DM; POTT CA; FURTANI PR; LIMONTA CR. 2005. Produtividade e qualidade de frutos de cultivares de tomateiro fertirrigado com cloreto e sulfato de potássio. Revista de Ciências Agroveterinárias. 4: p. 17-24.

FILGUEIRA FAR. 2000. Novo manual de olericultura. Viçosa: UFV, 402p.

GONÇALVES JLM; MENDES KCFS; SASAKI CM. 2001. Mineralização de nitrogênio em ecossistemas florestais naturais e implantados do Estado de São Paulo. Revista Brasileira de Ciência do Solo 25: 601-616.

LEAL FR; SANTOS VB; SALVIANO AAC. 2000. Sistemas de condução e aplicação de cal extinta na cultura do maxixe. Horticultura Brasileira 18: 542-543. Suplemento.

LOPES, AS. 2004. Manual Internacional de fertilidade do solo. Piracicaba: POTAFOS. $177 \mathrm{p}$.

MALAVOLTA E. 1990. Pesquisa com nitrogênio no Brasil, passado, presente e perspectivas. In: SIMPÓSIO BRASILEIRO SOBRE NITROGÊNIO EM PLANTAS, Anais... Itaguaí:, Sociedade Brasileira de Fisiologia Vegetal. p. 89-177.

MALAVOLTA E; GOMES FP; ALCARDE JC. 2002. Adubos e Adubações. São Paulo:
Nobel. 200p.

MALAVOLTA E; VITTI GC; OLIVEIRA SA. 1997. Avaliação do estado nutricional das plantas. Piracicaba: POTAFOS. 319p.

MARTINS MAS. 1986. Maxixe (Cucumis anguria L) e seu cultivo em São Luís do Maranhão. São Luís: EMAPA (Documento, 8).

MELOAMT; TRANI PS. Maxixe In: MELOAMT; FURLANI AMC. 1998. Instruções agrícolas para as principais culturas econômicas. IAC. 393p. (Boletim técnico, 209).

MELO WJ; MARQUES MO. 2000. Potencial do lodo como fonte de nutrientes para as plantas. In: W BETTIOL; OA CAMARGO. Impacto ambiental do uso agrícola do lodo de esgoto. Jaguariúna: EMBRAPA Meio Ambiente. p.109-142.

MENDONÇA ATC; PEIXOTO N. 1991. Efeitos do espaçamento e de níveis de adubação em cultivares de batata-doce. Horticultura Brasileira 9: 80-82.

MODOLO V; COSTA CP. 2000. Caracterização de frutificação e ponto de colheita em maxixe. Horticultura Brasileira 18: 476-478. Suplemento.

MODOLO VA; COSTA CP. 2003. Maxixe: uma hortaliça de tripla forma de consumo. Piracicaba: ESALQ. 20 p (Série produtor Rural, 19).

NOVAIS RF; VICTOR HAV; BARROS NF; FONTES RLF; CANTARUTTI NEVES JCL. 2007. Fertilidade do solo. Viçosa: SBCS. $1017 \mathrm{p}$.

OLIVEIRA FC. 2001. Percolação de nitrato em Latossolo Amarelo Distrófico afetada pela aplicação de composto de lixo urbano e adubação mineral. Revista Brasileira de Ciência do Solo 25: 731-741.

OLIVEIRAAP; ALVES AU; DORNELAS CSM; SILVA JA; PORTO ML; ALVES AU. 2003. Rendimento de quiabo em função de doses de nitrogênio. Acta Scientiarum agronomy 25: 265-268.

PIMENTEL AAMP. 1985. Olericultura no Trópico úmido., São Paulo: Ceres. 321p.

RIBEIRO AC. 1996. Como evitar a perda do nitrogênio de adubo por volatilização. Boletim Informativo da SBCS. 21: 43-46.

ROBINSON RW; DECKER-WALTER DS. 1997. Cucurbits. New york: CAB International, 225p.

RODRIGUES MB; KIEHL JC. 1992. Distribuição da amônia proveniente da uréia aplicada ao solo. Revista Brasileira de Ciência do Solo 16: 403-408.

SANGOI L; ERNANI PR.; LECH VA; RAMPAZZO C. 2003. Lixiviação de nitrogênio afetada pela forma de aplicação da uréia e manejo dos restos culturais de aveia em dois solos com texturas contrastantes. Ciência Rural 33: 65-70.

SILVA MG; ARF O; SÁ ME; RODRIGUES RAF; BUZETTI S. 2004. Nitrogen fertilization and soil management of winter common bean crop. Scientia Agrícola 61: 307-312.

SILVA WLC; MAROUELLI WA; CARRIJO OA; FONTES RR; MORETTI CL. 2000. Fontes de nitrogênio para fertirrigação de pimentão em ambiente protegido via gotejamento. Horticultura Brasileira 18: 822823. Suplemento. 


\title{
Yield and quality of strawberry cultivars
}

\author{
Luis Eduardo C Antunes; Nara Cristina Ristow; Ana Cristina R Krolow; Sílvia Carpenedo; Carlos \\ Reisser Júnior \\ Embrapa Clima Temperado, C. Postal 403,96001-970 Pelotas-RS; luis.eduardo@cpact.embrapa.br
}

\begin{abstract}
The strawberry cultivation is an important economic activity in the Rio Grande do Sul State, Brazil, however the number of offered cultivars to the growers is reduced. The yield and quality of strawberry (Fragaria x ananassa Duch.) cultivars Camarosa, Galexia, Earlibrite, Festival, Plarionfre and Sabrosa was evaluated under the climatic conditions of Pelotas, Rio Grande do Sul State. We determined the number, mass, total soluble solids (TSS ${ }^{\circ}$ Brix), total titratable acidity (TTA), antocianin level and fresh fruits produced in an experimental unit. We also determined the production of fruits per hectare and per plant. The statistical design used in the experiment was of completely randomized blocks with 6 treatments (cultivars) and 4 replicates where the experimental unit was composed of 8 plants. The harvest began in the first half of August, extending to the second half of December, totalling 20 weeks. Plarionfre, Earlibrite and Festival cultivars showed higher production from the first half of October until the end of the first half of November. Camarosa reached higher productivity, mass of plants and fruit weight. There were no differences between the evaluated cultivars in the levels of TSS, antocianin, ATT and $\mathrm{pH}$ during the period of evaluation.
\end{abstract}

Keywords: Fragaria $x$ ananassa Duch., productive performance, adaptation.

\section{RESUMO}

\section{Produtividade e qualidade de cultivares de morangueiro}

No estado do Rio Grande do Sul o cultivo do morangueiro é uma atividade econômica importante, entretanto o número de cultivares disponíveis no mercado é bastante reduzido. Assim buscou-se com este trabalho avaliar a produtividade e a qualidade dos frutos de cultivares de morangueiro (Fragaria x ananassa Duch.), Camarosa, Galexia, Earlibrite, Festival, Plarionfre e Sabrosa, cultivados nas condições climáticas e de solo do município de Pelotas, RS. Determinaram-se, o número, massa fresca e teor de sólidos solúveis totais (SST) (expresso em ${ }^{\circ}$ Brix), acidez total titulável (ATT) (expresso em \% de ácido cítrico) e teor de antocianinas, dos frutos produzidos por unidade experimental. Determinou-se ainda a produção acumulada de frutos (transformado em \% a cada 15 dias de colheita), produção por hectare e por planta. O experimento foi delineado em blocos inteiramente casualizados, com 6 tratamentos (cultivares) e 4 repetições onde a unidade experimental foi composta por 8 plantas. A colheita teve início na primeira quinzena de agosto, estendendo-se até a segunda quinzena de dezembro, totalizando 20 semanas. As cultivares Plarionfre, Earlibrite e Festival apresentaram maior produtividade acumulada a partir da primeira quinzena de outubro até o final da primeira quinzena de novembro, período este de maior percentual acumulado de colheita durante toda a safra. A cultivar Camarosa proporcionou maior produtividade, maior massa de fruto por planta e fruto com maior peso. Não houve diferenças para os teores de SST, pH, ATT e teores de antocianinas no decorrer de todo o período de avaliação.

Palavras-chave: Fragaria x ananassa Duch., desempenho produtivo, adaptação.

\section{(Recebido para publicação em 5 de janeiro de 2009; aceito em 3 de fevereiro de 2010)} (Received on January 5, 2009; accepted on February 3, 2010)

Cultivated strawberries belong to the family Rosaceae, in the genus Fragaria and to the species Fragaria x ananassa Duch. (Resende et al., 1999). The hybrid Fragaria x ananassa Duch. ex Rozier, resulting from natural hybridization between the species $F$. chiloensis and $F$. virginiana, is currently the most cultivated (Silva et al., 2007). The large variability between the species that compose the genetic base of Fragaria $\mathrm{x}$ ananassa allows a greater range of adaptation and quality of commercial strawberry cultivars (Passos, 1999; Santos \& Medeiros, 2003).

Large oscillations have occurred in production volume and in the area planted with strawberries in the last eight years (Kirschbaum \& Hancock, 2000; Antunes \& Reisser Junior, 2007) in the growing regions of Brazil, as a result of climatic variations, plant health problems, the market (Resende, 1999), and new cultivation techniques (Bortolozzo, 2006; Calvete et al., 2007).

The introduction of new strawberry cultivars is important since there is a natural substitution of the current varietal pattern with the adoption of cultivars with better characteristics of adaptation and management and in relation to qualitative aspects, which offer the farmers plants with better productive and qualitative characteristics and economic benefits. Genetic improvement programs concentrate their efforts in the obtaining of cultivars with plants that are easy to handle (small and erect), resistant to pests and diseases, high yielding and early, bearing big fruits with a good appearance and sweetness (Rios, 2007).

The introduction of cultivars by companies that sell plants, is a common practice adopted in the country. Meanwhile, the evaluation of the agronomic performance of these cultivars occurs in a localized manner and in those regions of interest for these companies, which makes decision- 